International Journal of Pure and Applied Mathematics

Volume 98 No. 1 2015, 81-98

ISSN: $1311-8080$ (printed version); ISSN: 1314-3395 (on-line version)

url: http://www.ijpam.eu

doi: http://dx.doi.org/10.12732/ijpam.v98i1.8

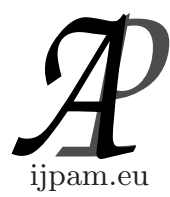

\title{
CONVOLUTIONS OF HYPER-ERLANG AND OF ERLANG DISTRIBUTIONS
}

\author{
Therrar Kadri ${ }^{1}$, Khaled Smaili ${ }^{2}$ \\ ${ }^{1}$ Department of Mathematics \\ Faculty of Sciences \\ Beirut Arab University \\ Beirut, LEBANON \\ ${ }^{2}$ Department of Applied Mathematics \\ Faculty of Sciences \\ Lebanese University \\ Zahle, LEBANON
}

\begin{abstract}
The sum of random variables are of interest in many areas of the sciences. In teletraffic analysis, the sum of Hyperexponential distribution is used as a model for the holding time distribution. Many authors examined this model and discussed its probability density function. In this paper, we consider the sum of independent Hyper-Erlang distributions. We showed that the probability density function of this distribution is related to probability density function of the sum of independent Erlang distributions- the Hypoexponential distribution. As a consequence, we find an exact closed expressions for the probability density function of both distribution, which are related to the Kummer confluent hypergeometric function.
\end{abstract}

AMS Subject Classification: 62E15, 60E10, 60E05

Key Words: convolution distribution, hyper-Erlang distribution, hypoexponential distribution, Erlang distribution, probability density, Bessel functions, confluent hypergeometric function

Received: August 29, 2014

(C) 2015 Academic Publications, Ltd.

${ }^{\S}$ Correspondence author url: www.acadpubl.eu 


\section{Introduction}

The sum of Random Variable (RV) or the convolution of RV plays an important role in modeling many events, see Ross [20] and Feller [9], as communications, computer science, teletraffic engineering (Trivedi [24], Jasiulewicz and Kordecki [12], Fang and Chlamtac [8]), Markov process (Kadri et al. [16], Jasiulewicz and Kordecki [12]), and reliability and performance evaluation (Trivedi [24], Jasiulewicz and Kordecki [12] and Bolsh [4]). Nadarajah [17] presented a review of some results on the sum of random variables.

In Traffic theory, one of the most important parameters in modeling a cellular network is the channel holding time distribution. Many authors showed that call holding time, cell residence time and channel holding time are no longer exponentially distributed, see (Barcelo and Jordan [3] and Orlik and Rappaport [18]. However, many authors proposed many other appropriate distributions models for the cell residence time and call holding time such as Gamma distribution by H. Zeng and Chlamtac [25] and Zeng et al. [26], Generalized Gamma distribution used by Zonoozi and Dassanayake [27] and Zonoozi et al. [28], Lognormal distribution by Jedrzycki and Leung [13], Erlang distribution by Fang et al. [7] and Glisic and Lorenzo ([10], Ch6. "Teletraffic Modeling and Analysis"), Hyper-Erlang distribution by Fang and Chlamtac [8], Fang et al. [7], and Glisic and Lorenzo [10], Hyperexponential distribution by Fang et al. [7], Coxian distribution by Soong and Barria [23], the sum of the Hyper-Exponential distribution (SOHYP) by Orlik and Rappaport, [18] and [19]. Eventually, the general Phase type (PH) distributions was used by Alfa et al. [1] and Christensen et al. [5]. We may also consider a more general appropriate distribution in traffic theory is the sum of independent Hyper-Erlang distribution. This distribution was not examined previously by any author before. However, its particular case, the sum of the Hyper-Exponential distribution (SOHYP), was proposed by some authors as the most appropriate distribution for call holding time as Orlik and Rappaport, [18] and Fang, [6].

In our paper, we consider the sum of two independent Hyper-Erlang random variables. We present the PDF of this distribution as a linear combination of the PDF of a special case of Hypoexponential random variable, the sum of two independent Erlang distributions. However, the later is related to the coefficient $A_{i j}$, the coefficient of the linear combination stated in Eq (6), discussed by Smaili et al in [21] and Kadri and Smaili in [15] and in both work $A_{i j}$ was given in recursive way. After some preliminaries of the subject in Section 2, in Section 3 , we find a closed exact expression of $A_{i j}$, and thus an exact expression of PDF for the sum of two independent Erlang distributions is determined and found 
to be a function of the Kummer confluent hypergeometric function, ${ }_{1} F_{1}[a, b, z]$. Next, in Section 4, we present all possible cases of the sum of two independent Hyper-Erlang distribution, and by using the results in Section 3, we find a closed expression of all these cases.

\section{Some Preliminaries}

\subsection{The Erlang Distribution}

The Erlang distribution is the sum of $k$ independent Exponential random variables each with same parameter $\alpha \in \mathbb{R}_{+}$. We write it as $\mathbb{E}_{\alpha}^{k} \sim \operatorname{Erl}(\alpha, k)$. The probability density function of $\mathbb{E}_{\alpha}^{k}$ is given as

$$
f_{\mathbb{E}_{\alpha}^{k}}(t)=\frac{(\alpha t)^{k-1} \alpha e^{-\alpha t}}{(k-1) !}
$$

if $t \geq 0$ and $f_{\mathbb{E}_{\alpha}^{k}}(t)=0$ if $t<0$. The Laplace transform of $f_{\mathbb{E}_{\alpha}^{k}}(t)$ is

$$
\mathcal{L}\left\{f_{\mathbb{E}_{\alpha}^{k}}(t)\right\}=\frac{\alpha^{k}}{(s+\alpha)^{k}}
$$

\subsection{The Hyper-Erlang Distribution}

The Hyper-Erlang distribution is the mixture of $m$ mutually independent Erlang distributions or parallel $m$-phase Erlang distribution weighted with the probabilities $\vec{p}=\left(p_{1}, p_{2}, \ldots, p_{m}\right)$ and each $i^{\text {th }}$ stage is the Erlang distribution $\mathbb{E}_{\alpha_{i}}^{k_{i}}$, for $1 \leq i \leq m$.

Let $X$ be a Hyper-Erlang distribution, then we write $X \sim H_{m}(\vec{p}, \vec{\alpha}, \vec{k})$ where $\vec{p}=\left(p_{1}, p_{2}, \ldots, p_{m}\right)$ are the weighted probabilities $p_{i} \geq 0$ and $\sum_{i=1}^{m} p_{i}=1$, $\vec{\alpha}=\left(\alpha_{1}, \alpha_{2}, \ldots, \alpha_{m}\right)$ are rate parameters, and $\vec{k}=\left(k_{1}, k_{2}, \ldots, k_{m}\right)$ are the shape parameter. The PDF of $X$ is given as

$$
f_{X}(t)=\sum_{i=1}^{m} p_{i} f_{\mathbb{E}_{\alpha_{i}}^{k_{i}}}(t)
$$


where $f_{\mathbb{E}_{\alpha_{i}}^{k_{i}}}(t)$ is the PDF of the Erlang distribution $\mathbb{E}_{\alpha_{i}}^{k_{i}}$ given in Eq (1). We also can conclude from Eq $(2)$ the Laplace transform of $f_{X}(t)$, given as

$$
\mathcal{L}\left\{f_{X}(t)\right\}=\sum_{j=1}^{m} \frac{p_{i} \alpha_{i}^{k_{i}}}{\left(s+\alpha_{i}\right)^{k_{i}}} .
$$

\subsection{Hypoexponential Distribution}

The Hypoexponential distribution is the distribution of the sum of $m \geq 2$ independent Exponential random variables presented by Smaili et al. [21]. The general case of the Hypoexponential distribution is when the $m$ exponential stages do not have to be distinct. This general case can be written as $\mathbb{H} \frac{\vec{k}}{\alpha}=\sum_{i=1}^{n} \sum_{j=1}^{k_{i}} X_{i j}$, where $X_{i j}$ is an Exponential random variable with parameter $\alpha_{i}, i=1,2, \ldots, n$, and written as $\mathbb{H} \frac{\vec{k}}{\vec{\alpha}} \sim \operatorname{Hypoexp}(\vec{\alpha}, \vec{k}), \vec{\alpha}=\left(\alpha_{1}, \alpha_{2}, \ldots, \alpha_{n}\right) \in \mathbb{R}_{+}^{n}$, where $\alpha_{i} \neq \alpha_{j}$ for all $i \neq j, \vec{k}=\left(k_{1}, k_{2}, \ldots, k_{n}\right) \in \mathbb{N}^{n}$ and $m=\sum_{i=1}^{n} k_{i}$. Since, the sum of independent Exponential RVs with same rate parameter is the Erlang distribution, then we may consider the Hypoexponential distribution to be the sum of $n$ independent different Erlang distribution, having each Erlang summand with rate parameter $\alpha_{i}$ and shape parameter $k_{i}, 1 \leq i \leq n$. Thus we may write

$$
\mathbb{H} \frac{\vec{k}}{\vec{\alpha}}=\sum_{i=1}^{n} \mathbb{E}_{\alpha_{i}}^{k_{i}}
$$

see Smaili et al. ([21], p.323-324). Moreover, they gave a modified expression for the PDF of the Hypoexponential random variable as

$$
f_{\mathbb{H} \frac{\vec{k}}{\vec{\alpha}}}(t)=\sum_{i=1}^{n} \sum_{j=1}^{k_{i}} A_{i j} f_{\mathbb{E}_{\alpha_{i}}^{j}}(t)
$$

where $f_{\mathbb{E}_{\alpha_{i}}^{j}}(t)$ is the PDF of the Erlang distribution $\mathbb{E}_{\alpha_{i}}^{j}$ given in Eq (1) for $1 \leq i \leq n, 1 \leq j \leq k_{i}$. However, Kadri and Smaili ([15], p.2667) gave an expression of $A_{i j}$ in a recursive way, stated in the following Lemma.

Lemma 1. Let $1 \leq i \leq n, 1 \leq j \leq k_{i}$. Then

$$
A_{i, k_{i}}=\prod_{j=1, j \neq i}^{n}\left(1-\frac{\alpha_{i}}{\alpha_{j}}\right)^{-k_{j}}
$$


and for $j=k_{i}-1$, down to 1 .

$$
A_{i j}=\frac{1}{k_{i}-j} \sum_{l=1}^{k_{i}-j}\left[\left(\sum_{p=1, p \neq i}^{n} k_{p}\left(1-\frac{\alpha_{p}}{\alpha_{i}}\right)^{-l}\right)^{\left.A_{i, j+l}\right]}\right.
$$

Also knowing that from Eq (5) that $\mathbb{H} \frac{\vec{k}}{\alpha}=\sum_{i=1}^{n} \mathbb{E}_{\alpha_{i}}^{k_{i}}$, and using Eq (2), we obtain the Laplace transform of $\mathbb{H} \frac{\vec{k}}{\alpha}$ as $\mathcal{L}\left\{f_{\mathbb{H} \frac{\vec{k}}{\alpha}}(t)\right\}=\prod_{i=1}^{n} \mathcal{L}\left\{f_{\mathbb{E}_{\alpha_{i}}^{k_{i}}}(t)\right\}=$ $\prod_{i=1}^{n}\left(\frac{\alpha_{i}^{k_{i}}}{\left(s+\alpha_{i}\right)^{k_{i}}}\right)$ from and thus the PDF of $\mathbb{H} \underset{\vec{\alpha}}{\vec{k}}$ can be obtained also as

$$
f_{\mathbb{H} \frac{\vec{k}}{\underline{\alpha}}}(t)=\mathcal{L}^{-1}\left\{\prod_{i=1}^{n}\left(\frac{\alpha_{i}^{k_{i}}}{\left(s+\alpha_{i}\right)^{k_{i}}}\right)\right\}
$$

In Section 3, we consider the case when $n=2$, that is the sum of two independent Erlang distribution. We like to highlight on this distribution. In this case we have $\mathbb{H}_{\frac{\vec{\alpha}}{\vec{k}}}^{\vec{k}} \triangleq \mathbb{H}_{\alpha_{1}, \alpha_{2}}^{k_{1}, k_{2}}=\mathbb{E}_{\alpha_{1}}^{k_{1}}+\mathbb{E}_{\alpha_{2}}^{k_{2}}$ and written as $\mathbb{H}_{\alpha_{1}, \alpha_{2}}^{k_{1}, k_{2}} \sim$ $\operatorname{Hypoexp}(\vec{\alpha}, \vec{k})$, where $\vec{\alpha}=\left(\alpha_{1}, \alpha_{2}\right) \in \mathbb{R}_{+}^{2}, \alpha_{1} \neq \alpha_{2}$ and $\vec{k}=\left(k_{1}, k_{2},\right) \in \mathbb{N}^{2}$. We conclude from (6) and (9) that the PDF of $\mathbb{H}_{\alpha_{1}, \alpha_{2}}^{k_{1}, k_{2}}$ is given in two expressions as

$$
\begin{aligned}
f_{\mathbb{H}_{\alpha_{1}, \alpha_{2}}^{k_{1}, k_{2}}}(t) & =\sum_{i=1}^{2} \sum_{j=1}^{k_{i}} A_{i j} f_{\mathbb{E}_{\alpha_{i}}^{j}}(t) \\
& =\mathcal{L}^{-1}\left\{\frac{\alpha_{1}^{k_{1}}}{\left(s+\alpha_{1}\right)^{k_{1}}} \times \frac{\alpha_{2}^{k_{2}}}{\left(s+\alpha_{2}\right)^{k_{2}}}\right\}
\end{aligned}
$$

In the next section, we consider this case and find an exact expression of the PDF of $\mathbb{H}_{\alpha_{1}, \alpha_{2}}^{k_{1}, k_{2}}$, which shall be used later in Section 4 to find the exact expression of the sum of two independent Hyper-Erlang distribution.

\section{The Exact Expression of Sum of Two Independent Erlang Distributions}

In this section, we given an exact closed expression of the PDF for the sum of two independent Erlang, $\mathbb{H}_{\alpha_{1}, \alpha_{2}}^{k_{1}, k_{2}}$. The result obtained shall be used in the next 
section to find a closed expression for the PDF of the sum of two Hyper-Erlang distributions.

From Eq (10), we have $f_{\mathbb{H}_{\alpha_{1}, \alpha_{2}}^{k_{1}, k_{2}}}(t)=\sum_{i=1}^{2} \sum_{j=1}^{k_{i}} A_{i j} f_{\mathbb{E}_{\alpha_{i}}^{j}}(t)$. Since $f_{\mathbb{E}_{\alpha_{i}}^{j}}(t)$ is given in Eq (1), we are left to find a closed expression of $A_{i j}, i=1,2$ and $1 \leq j \leq k_{i}$ defined recursively in Lemma 1 . We start by finding $A_{i j}$, then $f_{\mathbb{H}_{\alpha_{1}, \alpha_{2}}^{k_{1}, k_{2}}}(t)$ in the next two theorems..

Theorem 1. Let $\vec{\alpha}=\left(\alpha_{1}, \alpha_{2}\right), \vec{k}=\left(k_{1}, k_{2}\right)$, and suppose that $a=$ $\alpha_{1}+\alpha_{2}$ and $m=k_{1}+k_{2}$. Then

$$
A_{i j}=(-1)^{k_{i}-j}\left(\begin{array}{c}
m-j-1 \\
m-k_{i}-1
\end{array}\right) \frac{\alpha_{i}^{k_{i}-j}\left(a-\alpha_{i}\right)^{m-k_{i}}}{\left(a-2 \alpha_{i}\right)^{m-j}}, i=1,2 \text { and } j=1,2, \ldots, k_{i} \text {. }
$$

Note that $A_{1 j}=(-1)^{k_{1}-j}\left(\begin{array}{c}m-j-1 \\ k_{2}-1\end{array}\right) \frac{\alpha_{1}^{k_{1}-j} \alpha_{2}^{k_{2}}}{\left(\alpha_{2}-\alpha_{1}\right)^{m-j}}, j=1,2, \ldots, k_{1}$ and $A_{2 j}=$ $(-1)^{k_{2}-j} \times\left(\begin{array}{c}m-j-1 \\ k_{1}-1\end{array}\right) \frac{\alpha_{2}^{k_{2}-j} \alpha_{1}^{k_{1}}}{\left(\alpha_{1}-\alpha_{2}\right)^{m-j}}, j=1,2, \ldots, k_{2}$.

Proof. We start by taking $i=1$. We shall prove that

$$
A_{1 j}=(-1)^{k_{1}-j}\left(\begin{array}{c}
m-j-1 \\
k_{2}-1
\end{array}\right) \frac{\alpha_{1}^{k_{1}-j} \alpha_{2}^{k_{2}}}{\left(\alpha_{2}-\alpha_{1}\right)^{m-j}}, j=1,2, \ldots, k_{1}
$$

by down induction. We begin with $j=k_{1}$, From the expression of $A_{1, k_{1}}$ in Eq (7), we get $A_{1, k_{1}}=\prod_{j=1, j \neq i}^{2}\left(1-\frac{\alpha_{i}}{\alpha_{j}}\right)^{-k_{j}}=\left(\frac{\alpha_{2}}{\alpha_{2}-\alpha_{1}}\right)^{k_{2}}$. On the other hand, Eq (13) gives that $A_{1, k_{1}}=(-1)^{k_{1}-k_{1}}\left(\begin{array}{c}m-k_{1}-1 \\ k_{2}-1\end{array}\right) \frac{\alpha_{1}^{k_{1}-k_{1}} \alpha_{2}^{k_{2}}}{\left(\alpha_{2}-\alpha_{1}\right)^{m_{-}-k_{1}}}=\frac{\alpha_{2}^{k_{2}}}{\left(\alpha_{2}-\alpha_{1}\right)^{k_{2}}}$. Thus, for $j=k_{1}$, the expression is true. Suppose that the expression is true for $A_{1, j+1}$, we want to prove its true for $A_{1 j}$. From Eq (8), we have

$$
\begin{aligned}
A_{1 j} & =\frac{1}{k_{1}-j} \sum_{l=1}^{k_{1}-j}\left[\left(\sum_{p=1, p \neq 1}^{2} k_{p}\left(1-\frac{\alpha_{p}}{\alpha_{1}}\right)^{-l}\right)^{A_{1, j+l}}\right] \\
& =\frac{k_{2}}{k_{1}-j} \sum_{l=1}^{k_{1}-j}\left[\left(\left(1-\frac{\alpha_{2}}{\alpha_{1}}\right)^{-l}\right) A_{1, j+l}\right]
\end{aligned}
$$

But $A_{1, j+l}=(-1)^{k_{1}-j-l}\left(\begin{array}{c}m-j-l-1 \\ k_{2}-1\end{array}\right) \frac{\alpha_{1}^{k_{1}-j-l} \alpha_{2}^{k_{2}}}{\left(\alpha_{2}-\alpha_{1}\right)^{m-j-l}}$, obtained from Eq (13), thus

$$
A_{1 j}=\frac{k_{2}}{k_{1}-j} \sum_{l=1}^{k_{1}-j}\left[(-1)^{k_{1}-j}\left(\begin{array}{c}
m-j-l-1 \\
k_{2}-1
\end{array}\right) \frac{\alpha_{1}^{k_{1}-j} \alpha_{2}^{k_{2}}}{\left(\alpha_{2}-\alpha_{1}\right)^{m-j}}\right]
$$




$$
=\frac{(-1)^{k_{1}-j} k_{2} \alpha_{1}^{k_{1}-j} \alpha_{2}^{k_{2}}}{\left(k_{1}-j\right)\left(\alpha_{2}-\alpha_{1}\right)^{m-j}} \sum_{l=1}^{k_{1}-j}\left(\begin{array}{c}
m-j-l-1 \\
k_{2}-1
\end{array}\right)
$$

Next, we find a simplified expression of $\sum_{l=1}^{k_{1}-j}\left(\begin{array}{c}m-j-l-1 \\ k_{2}-1\end{array}\right)$. First we can rewrite it $\operatorname{as} \sum_{l=1}^{k_{1}-j}\left(\begin{array}{c}m-j-l-1 \\ k_{2}-1\end{array}\right)=\sum_{l=1}^{k_{1}-j}\left(\begin{array}{c}k_{1}+k_{2}-j-l-1 \\ k_{2}-1\end{array}\right)=\sum_{l=0}^{k_{1}-j-1}\left(\begin{array}{c}k_{1}+k_{2}-j-l-2 \\ k_{2}-1\end{array}\right)$. We shall use the northwest-diagonal sum property of binomial coefficient that is $\sum_{l=0}^{r}\left(\begin{array}{l}n-l \\ r-l\end{array}\right)=$ $\left(\begin{array}{c}n+1 \\ r\end{array}\right)$, see (Gross [11], p.223). Writing $\left(\begin{array}{c}n-l \\ r-l\end{array}\right)=\left(\begin{array}{c}n-l \\ n-r\end{array}\right)$, then this property can be written as $\sum_{l=0}^{r}\left(\begin{array}{c}n-l \\ n-r\end{array}\right)=\left(\begin{array}{c}n+1 \\ r\end{array}\right)$. Set $r=k_{1}-j-1$ and $n=k_{1}+k_{2}-j-2$, we obtain that $\sum_{l=0}^{k_{1}-j-1}\left(\begin{array}{c}k_{1}+k_{2}-j-l-2 \\ n-r\end{array}\right)=\left(\begin{array}{c}k_{1}+k_{2}-j-1 \\ k_{1}-j-1\end{array}\right)=\left(\begin{array}{c}k_{1}+k_{2}-j-1 \\ k_{2}\end{array}\right)$, also using absorption property of binomial $\left(\begin{array}{l}n \\ r\end{array}\right)=\frac{n}{r}\left(\begin{array}{c}n-l \\ r-l\end{array}\right)$, see (Gross [11], p.224), we obtain that $\left(\begin{array}{c}k_{1}+k_{2}-j-1 \\ k_{2}\end{array}\right)=\frac{k_{1}+k_{2}-j-1}{k_{2}}\left(\begin{array}{c}k_{1}+k_{2}-j-2 \\ k_{2}-1\end{array}\right)$. Therefore, as a conclusion

$$
\sum_{l=1}^{k_{1}-j}\left(\begin{array}{c}
m-j-l-1 \\
k_{2}-1
\end{array}\right)=\frac{m-j-1}{k_{2}}\left(\begin{array}{c}
m-j-2 \\
k_{2}-1
\end{array}\right)
$$

Now, by substituting (15) in (14), we get

$$
\begin{aligned}
A_{1 j} & =(-1)^{k_{1}-j} \frac{m-j-1}{k_{2}} \times \frac{k_{2}}{k_{1}-j} \times\left(\begin{array}{c}
m-j-2 \\
k_{2}-1
\end{array}\right) \frac{\alpha_{1}^{k_{1}-j} \alpha_{2}^{k_{2}}}{\left(\alpha_{2}-\alpha_{1}\right)^{m-j}} \\
& =(-1)^{k_{1}-j}\left(\begin{array}{c}
m-j-1 \\
k_{2}-1
\end{array}\right) \frac{\alpha_{1}^{k_{1}-j} \alpha_{2}^{k_{2}}}{\left(\alpha_{2}-\alpha_{1}\right)^{m-j}}
\end{aligned}
$$

Thus we proved for $j=k_{1}$ down to 1 that $A_{1 j}=(-1)^{k_{1}-j}\left(\begin{array}{c}m-j-1 \\ k_{2}-1\end{array}\right) \frac{\alpha_{1}^{k_{1}-j} \alpha_{2}^{k_{2}}}{\left(\alpha_{2}-\alpha_{1}\right)^{m-j}}$.

On the other hand, for $i=2$, and in the same procedure, since $\alpha_{1}, \alpha_{2}$ and $k_{1}, k_{2}$ are symmetric, we obtain that $A_{2 j}=(-1)^{k_{2}-j}\left(\begin{array}{c}m-j-1 \\ k_{1}-1\end{array}\right) \frac{\alpha_{2}^{k_{2}-j} \alpha_{1}^{k_{1}}}{\left(\alpha_{1}-\alpha_{2}\right)^{m-j}}$, $j=1,2, \ldots, k_{2}$, that verifies the expression (12) for $j=2$.

Next, we give the exact closed expression of the sum of two independent Erlang distribution, $\mathbb{H}_{\alpha_{1}, \alpha_{2}}^{k_{1}, k_{2}}$.

Theorem 2. Let $\mathbb{H}_{\alpha_{1}, \alpha_{2}}^{k_{1}, k_{2}}$ be a sum of two Erlang distributions. Then the $P D F$ of $\mathbb{H}_{\alpha_{1}, \alpha_{2}}^{k_{1}, k_{2}}$ is given as

$$
f_{\mathbb{H}_{\alpha_{1}, \alpha_{2}}^{k_{1}, k_{2}}}(t)=\frac{(-1)^{k_{1}+1} \alpha_{1}^{k_{1}} \alpha_{2}^{k_{2}}\left(\begin{array}{c}
k_{1}+k_{2}-2 \\
k_{2}-1
\end{array}\right)}{\left(\alpha_{2}-\alpha_{1}\right)^{k_{1}+k_{2}-1}}\left(e_{1}^{-\alpha_{1} t}{ }_{1} F_{1}\left[1-k_{1},-k_{1}-k_{2}+2,\left(\alpha_{1}-\alpha_{2}\right) t\right]\right.
$$




$$
\left.-e^{-\alpha_{2} t}{ }_{1} F_{1}\left[1-k_{2},-k_{1}-k_{2}+2,\left(\alpha_{2}-\alpha_{1}\right) t\right]\right),
$$

where ${ }_{1} F_{1}[a, b, z]$ is the Kummer confluent hypergeometric function defined as ${ }_{1} F_{1}[a, b, z]=\sum_{j=0}^{-a} \frac{(a)_{j}(z)^{j}}{(b)_{j} \times j !}$ for $-a,-b \in \mathbb{N}$ and $b>a$ and $(a)_{j}$ is the rising factorial, see Wolfram Function site, [29].

Proof. From Eq (10), the PDF of $\mathbb{H}_{\alpha_{1}, \alpha_{2}}^{k_{1}, k_{2}}$ is

$$
f_{\mathbb{H}_{\alpha_{1}, \alpha_{2}}^{k_{1}, k_{2}}}(t)=\sum_{i=1}^{2} \sum_{j=1}^{k_{i}} A_{i j} f_{\mathbb{E}_{\alpha_{i}}^{j}}(t)=\sum_{j=1}^{k_{1}} A_{1 j} f_{\mathbb{E}_{\alpha_{1}}^{j}}(t)+\sum_{j=1}^{k_{2}} A_{2 j} f_{\mathbb{E}_{\alpha_{2}}^{j}}(t) .
$$

However, from Theorem 1, we have

$$
\begin{gathered}
A_{i j}=(-1)^{k_{i}-j}\left(\begin{array}{c}
m-j-1 \\
m-k_{i}-1
\end{array}\right) \frac{\alpha_{i}^{k_{i}-j}\left(a-\alpha_{i}\right)^{m-k_{i}}}{\left(a-2 \alpha_{i}\right)^{m-j}}, \quad j=1,2, \ldots, k_{i}, \quad i=1,2, \\
m=k_{1}+k_{2}, \quad a=\alpha_{1}+\alpha_{2},
\end{gathered}
$$

thus

$$
A_{1 j}=(-1)^{k_{1}-j}\left(\begin{array}{c}
m-j-1 \\
k_{2}-1
\end{array}\right) \frac{\alpha_{1}^{k_{1}-j} \alpha_{2}^{k_{2}}}{\left(\alpha_{2}-\alpha_{1}\right)^{m-j}}, \quad j=1,2, \ldots, k_{1}
$$

and

$$
A_{2 j}=(-1)^{k_{2}-j}\left(\begin{array}{c}
m-j-1 \\
k_{1}-1
\end{array}\right) \frac{\alpha_{2}^{k_{2}-j} \alpha_{1}^{k_{1}}}{\left(\alpha_{1}-\alpha_{2}\right)^{m-j}}, \quad j=1,2, \ldots, k_{2},
$$

and $f_{\mathbb{E}_{\alpha_{i}}^{j}}(t), i=1,2$ from $\mathrm{Eq}(1)$. Thus we obtain that

$$
\begin{aligned}
& f_{\mathbb{H}_{\alpha_{1}, \alpha_{2}}^{k_{1}, k_{2}}}(t) \\
= & \sum_{j=1}^{k_{1}}\left(\begin{array}{c}
m-j-1 \\
k_{2}-1
\end{array}\right) \frac{(-1)^{k_{1}-j} \alpha_{1}^{k_{1}} \alpha_{2}^{k_{2}} e^{-\alpha_{1} t} t^{j-1}}{\left(\alpha_{2}-\alpha_{1}\right)^{m-j}(j-1) !}+\sum_{j=1}^{k_{2}}\left(\begin{array}{c}
m-j-1 \\
k_{1}-1
\end{array}\right) \frac{(-1)^{k_{2}-j} \alpha_{1}^{k_{1}} \alpha_{2}^{k_{2}} e^{-\alpha_{2} t} t^{j-1}}{\left(\alpha_{1}-\alpha_{2}\right)^{m-j}(j-1) !} .
\end{aligned}
$$

Writing $(-1)^{k_{2}-j}=(-1)^{m-j}(-1)^{k_{1}}$ we get

$$
\begin{aligned}
f_{\mathbb{H}_{\alpha_{1}, \alpha_{2}}^{k_{1}, k_{2}}}(t)= & \frac{(-1)^{k_{1}} \alpha_{1}^{k_{1}} \alpha_{2}^{k_{2}}}{\left(\alpha_{2}-\alpha_{1}\right)^{m}}\left[e^{-\alpha_{1} t} \sum_{j=1}^{k_{1}}\left(\begin{array}{c}
m-j-1 \\
k_{2}-1
\end{array}\right) \frac{\left(\alpha_{1}-\alpha_{2}\right)^{j} t^{j-1}}{(j-1) !}+\right. \\
& e^{-\alpha_{2} t} \sum_{j=1}^{k_{2}}\left(\begin{array}{c}
m-j-1 \\
k_{1}-1
\end{array}\right) \frac{\left(\alpha_{2}-\alpha_{1}\right)^{j} t^{j-1}}{(j-1) !}
\end{aligned}
$$


Next, we shall prove that

$$
\sum_{j=1}^{k_{1}}\left(\begin{array}{c}
m-j-1 \\
k_{2}-1
\end{array}\right) \frac{b^{j} t^{j-1}}{(j-1) !}=b\left(\begin{array}{c}
m-2 \\
k_{2}-1
\end{array}\right){ }_{1} F_{1}\left[1-k_{1},-m+2, b t\right]
$$

taking $b=\alpha_{1}-\alpha_{2}$. Now, since $1-k_{1}, 2-m$ are nonpositive integers and $m-2>k_{1}-1$, then ${ }_{1} F_{1}\left[1-k_{1},-m+2, b t\right]$ is defined as

$$
{ }_{1} F_{1}\left[1-k_{1},-m+2, b t\right]=\sum_{j=0}^{k_{1}-1} \frac{\left(1-k_{1}\right)_{j}(b t)^{j}}{(m-2)_{j} \times j !}=\sum_{j=1}^{k_{1}} \frac{\left(1-k_{1}\right)_{j-1}(b t)^{j-1}}{(m-2)_{j-1}(j-1) !} \text {. Starting }
$$
with $b\left(\begin{array}{c}m-2 \\ k_{2}-1\end{array}\right){ }_{1} F_{1}\left[1-k_{1},-m+2, b t\right]$, we get

$$
b\left(\begin{array}{c}
m-2 \\
k_{2}-1
\end{array}\right)_{1} F_{1}\left[1-k_{1},-m+2, b t\right]=b \frac{(m-2) !}{\left(k_{2}-1\right) !\left(k_{1}-1\right) !} \sum_{j=1}^{k_{1}} \frac{\left(1-k_{1}\right)_{j-1}(b t)^{j-1}}{(2-m)_{j-1} \times(j-1) !}
$$

However, when $x \in \mathbb{N}$ and $x \geq n$ the rising factorial $(-x)_{n}$ can be written as $(-x)_{n}=(-1)^{n} \frac{x !}{(x-n) !}$, see (Andrews [2], p.358). Thus, we get $\left(1-k_{1}\right)_{j-1}=$ $(-1)^{j-1} \frac{\left(k_{1}-1\right) !}{\left(k_{1}-j\right) !}$ and $(2-m)_{j-1}=(-1)^{j-1} \frac{(m-2) !}{(m-j-1) !}$ after substituting in (19) we obtain

$$
\begin{aligned}
b\left(\begin{array}{c}
m-2 \\
k_{2}-1
\end{array}\right){ }_{1} F_{1}\left[1-k_{1},-m+2, b t\right] & =b \frac{(m-2) !}{\left(k_{2}-1\right) !\left(k_{1}-1\right) !} \sum_{j=1}^{k_{1}} \frac{\frac{\left(k_{1}-1\right) !}{\left(k_{1}-j\right) !}(b t)^{j-1}}{\frac{(m-2) !}{(m-j-1) !} \times(j-1) !} \\
& =\frac{b}{\left(k_{2}-1\right) !} \sum_{j=1}^{k_{1}} \frac{(m-j-1) !(b t)^{j-1}}{\left(k_{1}-j\right) ! \times(j-1) !} \\
& =\sum_{j=1}^{k_{1}}\left(\begin{array}{c}
m-j-1 \\
k_{2}-1
\end{array}\right) \frac{b^{j} t^{j-1}}{(j-1) !}
\end{aligned}
$$

In a similar way, we obtain that

$$
\sum_{j=1}^{k_{2}}\left(\begin{array}{c}
m-j-1 \\
k_{1}-1
\end{array}\right) \frac{c^{j} t^{j-1}}{(j-1) !}=c\left(\begin{array}{c}
m-2 \\
k_{1}-1
\end{array}\right){ }_{1} F_{1}\left[1-k_{2},-m+2, c t\right]
$$

with $c=\alpha_{2}-\alpha_{1}$. After substituting (18) and (20) in the obtained expression of the $\mathrm{PDF}$ in $\mathrm{Eq}(17)$, we get

$$
\begin{aligned}
& f_{\mathbb{H}_{\mathbb{H}_{1}, \alpha_{2}}^{k_{1}, k_{2}}}(t) \\
& \quad=\frac{(-1)^{k_{1}} \alpha_{1}^{k_{1}} \alpha_{2}^{k_{2}}}{\left(\alpha_{2}-\alpha_{1}\right)^{m}}\left[e^{-\alpha_{1} t}\left(\alpha_{1}-\alpha_{2}\right)\left(\begin{array}{c}
m-2 \\
k_{2}-1
\end{array}\right){ }_{1} F_{1}\left[1-k_{1},-m+2,\left(\alpha_{1}-\alpha_{2}\right) t\right]\right.
\end{aligned}
$$




$$
+e^{-\alpha_{2} t}\left(\alpha_{2}-\alpha_{1}\right)\left(\begin{array}{c}
m-2 \\
k_{1}-1
\end{array}\right){ }_{1} F_{1}\left[1-k_{2},-m+2,\left(\alpha_{2}-\alpha_{1}\right) t\right]
$$

$\operatorname{But}\left(\begin{array}{l}m-2 \\ k_{2}-1\end{array}\right)=\left(\begin{array}{c}m-2 \\ k_{1}-1\end{array}\right)$, then

$$
\begin{array}{r}
f_{\mathbb{H}_{\alpha_{1}, \alpha_{2}}^{k_{1}, k_{2}}}(t)=\frac{(-1)^{k_{1}+1} \alpha_{1}^{k_{1}} \alpha_{2}^{k_{2}}\left(\begin{array}{l}
m-2 \\
k_{2}-1
\end{array}\right)}{\left(\alpha_{2}-\alpha_{1}\right)^{m-1}}\left[e ^ { - \alpha _ { 1 } t } { } _ { 1 } F _ { 1 } \left[1-k_{1},-m+2,\left(\alpha_{1}-\alpha_{2}\right) t\right.\right. \\
-e^{-\alpha_{2} t}{ }_{1} F_{1}\left[1-k_{2},-m+2,\left(\alpha_{2}-\alpha_{1}\right) t\right] .
\end{array}
$$

We may give from our above results a new important result in the Laplace transform theory by finding an exact form of the Laplace inverse transform for the product of any two partial fractions with multiple roots. The expression is obtained directly from Eq (10) which shows that the PDF of sum of two Erlang distributions is the Laplace inverse of the latter. We state it in the following Proposition.

Proposition 1. Let $\left(\alpha_{1}, \alpha_{2}\right) \in \mathbb{C}^{2}, \alpha_{1} \neq \alpha_{2},\left(k_{1}, k_{2}\right) \in \mathbb{N}^{2}$ and $m=k_{1}+k_{2}$

$$
\begin{aligned}
& \mathcal{L}^{-1}\left\{\frac{1}{\left(s+\alpha_{1}\right)^{k_{1}}} \times \frac{1}{\left(s+\alpha_{2}\right)^{k_{2}}}\right\} \\
&=\frac{(-1)^{k_{1}+1}\left(\begin{array}{l}
m-2 \\
k_{2}-1
\end{array}\right)}{\left(\alpha_{2}-\alpha_{1}\right)^{m-1}}\left[e ^ { - \alpha _ { 1 } t } { } _ { 1 } F _ { 1 } \left[1-k_{1},-k_{1}-k_{2}+2,\left(\alpha_{1}-\alpha_{2}\right) t\right.\right. \\
& \quad-e^{-\alpha_{2} t}{ }_{1} F_{1}\left[1-k_{2},-k_{1}-k_{2}+2,\left(\alpha_{2}-\alpha_{1}\right) t\right]
\end{aligned}
$$

Proof. From Eq (11) and Theorem 2 the PDF of $\mathbb{H}_{\alpha_{1}, \alpha_{2}}^{k_{1}, k_{2}}$ is given in two expressions as

$$
\begin{aligned}
& f_{\mathbb{H}_{\alpha_{1}, \alpha_{2}}^{k_{1}, k_{2}}}(t)=\mathcal{L}^{-1}\left\{\frac{\alpha_{1}^{k_{1}}}{\left(s+\alpha_{1}\right)^{k_{1}}} \times \frac{\alpha_{2}^{k_{2}}}{\left(s+\alpha_{2}\right)^{k_{2}}}\right\} \\
= & \frac{(-1)^{k_{1}+1} \alpha_{1}^{k_{1}} \alpha_{2}^{k_{2}}\left(\begin{array}{c}
k_{1}+k_{2}-2 \\
k_{2}-1
\end{array}\right)}{\left(\alpha_{2}-\alpha_{1}\right)^{d_{1}+d_{2}-1}}\left(e_{1}^{-\alpha_{1} t} F_{1}\left[1-k_{1},-k_{1}-k_{2}+2,\left(\alpha_{1}-\alpha_{2}\right) t\right]-e^{-\alpha_{2} t}{ }_{1} F_{1}\right. \\
& {\left.\left[1-k_{2},-k_{1}-k_{2}+2,\left(\alpha_{2}-\alpha_{1}\right) t\right]\right) . }
\end{aligned}
$$

After canceling $\alpha_{1}^{k_{1}} \alpha_{2}^{k_{2}}$ from both sides, we obtain the result. Moreover, $\alpha_{1}$ and $\alpha_{2}$ can be taken complex numbers, since the variable $z$ in the Kummer confluent hypergeometric function, ${ }_{1} F_{1}[a, b, z]$, can be complex and the steps used in the proof of Theorem 2 are unaltered. 


\section{The Exact Expression of Sum of Two Independent Hyper-Erlang Distribution}

In this section, we find a closed expression of the sum of two independent HyperErlang distributions. This closed expression is related directly to the PDF of the sum of two Erlang distributions, that is solved completely in the previous section in Theorem 2. Moreover, we consider all possible cases of the problem.

We suppose that $X$ and $Y$ are two independent Hyper-Erlang distributions. Let $X \sim H_{m}(\vec{p}, \vec{\alpha}, \vec{k}), \vec{p}=\left(p_{1}, p_{2}, \ldots, p_{m}\right), \vec{\alpha}=\left(\alpha_{1}, \alpha_{2}, \ldots, \alpha_{m}\right)$, and $\vec{k}=$ $\left(k_{1}, k_{2}, \ldots, k_{m}\right)$ and $Y \sim H_{n}(\vec{q}, \vec{\beta}, \vec{l}), \vec{q}=\left(q_{1}, q_{2}, \ldots, q_{n}\right), \vec{\beta}=\left(\beta_{1}, \beta_{2}, \ldots, \beta_{n}\right)$, and $\vec{l}=\left(l_{1}, l_{2}, \ldots, l_{n}\right)$, for all $1 \leq i \leq m$ and $1 \leq j \leq n$. From Eq (3) the PDFs of $X$ and $Y$ are $f_{X}(t)=\sum_{i=1}^{m} p_{i} f_{\mathbb{E}_{\alpha_{i}}^{k_{i}}}(t)$ and $f_{Y}(t)=\sum_{j=1}^{n} q_{j} f_{\mathbb{E}_{\beta_{j}}^{l_{j}}}(t)$.

We consider the following cases.

1. When the rate parameters are equal, $\vec{\alpha}=\vec{\beta}$ (particularly, the case of identical RVs).

2. When the rate parameters are different $\alpha_{i} \neq \beta_{j}$, for all $1 \leq i \leq m$ and $1 \leq j \leq n$.

3. When the rate parameters are not all different (the general case).

\subsection{Case of Identical Rate Parameters}

In this section, we suppose that $X$ and $Y$ are two independent Hyper-Erlang distribution where their rate parameters are identical. We take $X \sim H_{m}(\vec{p}, \vec{\alpha}, \vec{k})$ and $Y \sim H_{m}(\vec{q}, \vec{\alpha}, \vec{l})$, with $\vec{p}=\left(p_{1}, p_{2}, \ldots, p_{m}\right), \vec{q}=\left(q_{1}, q_{2}, \ldots, q_{m}\right) \vec{\alpha}=$ $\left(\alpha_{1}, \alpha_{2}, \ldots, \alpha_{m}\right), \vec{k}=\left(k_{1}, k_{2}, \ldots, k_{m}\right)$ and $\vec{l}=\left(l_{1}, l_{2}, \ldots, l_{m}\right)$. From Eq (3) the PDFs of $X$ and $Y$ are

$$
f_{X}(t)=\sum_{i=1}^{m} p_{i} f_{\mathbb{E}_{\alpha_{i}}^{k_{i}}}(t) \text { and } f_{Y}(t)=\sum_{j=1}^{m} q_{j} f_{\mathbb{E}_{\alpha_{j}}^{l_{i}}}(t)
$$

Theorem 3. Let $X$ and $Y$ be two independent random variables distributed according to (21). Then the PDF of $S=X+Y$ is given by

$$
f_{S}(t)=\sum_{i=1}^{m} p_{i} q_{i} f_{\mathbb{E}_{\alpha_{i}}^{k_{i}}}(t)+\sum_{i=1}^{m} \sum_{j=1, j \neq i}^{m} p_{i} q_{j} f_{\mathbb{H}_{\alpha_{i}, \alpha_{j}}^{k_{i}, l_{j}}}(t)
$$

where $f_{\mathbb{H}_{\alpha_{i}, \alpha_{j}}^{k_{i}, l_{j}}}(t)$ is given in Eq (16) and $f_{\mathbb{E}_{\alpha_{i}}^{k_{i}}}(t)$ is given in Eq (1). 
Proof. Since $X$ and $Y$ are independent, then $\mathcal{L}\left\{f_{S}(t)\right\}=\mathcal{L}\left\{f_{X}(t)\right\} \mathcal{L}\left\{f_{Y}(t)\right\}$. However, the Laplace transform of the Hyper-Erlang is given in Eq 4, then $\mathcal{L}\left\{f_{S}(t)\right\}=\sum_{i=1}^{m} \sum_{j=1}^{m} p_{i} q_{j} \frac{\alpha_{i}^{k_{i}}}{\left(s+\alpha_{i}\right)^{k_{i}}} \times \frac{\alpha_{j}^{l_{j}}}{\left(s+\alpha_{j}\right)^{l_{j}}}$ and we may conclude that $f_{S}(t)=$ $\sum_{i=1}^{m} \sum_{j=1}^{m} p_{i} q_{j} \mathcal{L}^{-1}\left\{\frac{\alpha_{i}^{k_{i}}}{\left(s+\alpha_{i}\right)^{k_{i}}} \times \frac{\alpha_{j}^{l_{j}}}{\left(s+\alpha_{j}\right)^{l_{j}}}\right\}$. By separating the expression $\frac{\alpha_{i}^{k_{i}}}{\left(s+\alpha_{i}\right)^{k_{i}}} \times$ $\frac{\alpha_{j}^{k_{j}}}{\left(s+\alpha_{j}\right)^{k_{j}}}$ into distinct factors, we may use Eq (10) to find the Laplace inverse. Thus we take out the part when $i=j$, to leave the part when $i \neq j$, Hence, we obtain that

$$
\begin{aligned}
f_{S}(t)=\sum_{i=1}^{m} p_{i} q_{i} \mathcal{L}^{-1}\left\{\frac{\alpha_{i}^{k_{i}+l_{i}}}{\left(s+\alpha_{i}\right)^{k_{i}+l_{i}}}\right\} & \\
& \quad+\sum_{i=1}^{m} \sum_{j=1, j \neq i}^{m} p_{i} q_{j} \mathcal{L}^{-1}\left\{\frac{\alpha_{i}^{k_{i}}}{\left(s+\alpha_{i}\right)^{k_{i}}} \times \frac{\alpha_{j}^{l_{j}}}{\left(s+\alpha_{j}\right)^{l_{j}}}\right\}
\end{aligned}
$$

However, from $\operatorname{Eq}(2), \mathcal{L}^{-1}\left\{\frac{\alpha_{i}^{k_{i}+l_{i}}}{\left(s+\alpha_{i}\right)^{k_{i}+l_{i}}}\right\}=f_{\mathbb{E}_{\alpha_{i}}^{k_{i}+l_{i}}}(t)$, also from $\operatorname{Eq}(10)$, we obtain that $\mathcal{L}^{-1}\left\{\frac{\alpha_{i}^{k_{i}}}{\left(s+\alpha_{i}\right)^{k_{i}}} \times \frac{\alpha_{j}^{l_{j}}}{\left(s+\alpha_{j}\right)^{l_{j}}}\right\}=f_{\mathbb{H}_{\alpha_{i}, \alpha_{i}, l_{j}}}(t)$, since $i \neq j$. Therefore, we obtain the required PDF expression

Next, we consider a particular case of the above case is when the two RVs are identical.

Corollary 4. Let $X$ and $Y$ be two identical independent Hyper-Erlang distribution. Let $X$ and $Y$ follow the distribution $H_{m}(\vec{p}, \vec{\alpha}, \vec{k})$, with $\vec{p}=$ $\left(p_{1}, p_{2}, \ldots, p_{m}\right), \vec{\alpha}=\left(\alpha_{1}, \alpha_{2}, \ldots, \alpha_{m}\right)$, and $\vec{k}=\left(k_{1}, k_{2}, \ldots, k_{m}\right)$. Then the PDF of $S=X+Y$ is

$$
f_{S}(t)=\sum_{i=1}^{m} p_{i}^{2} f_{\mathbb{E}_{\alpha_{i}}^{2 k_{i}}}(t)+\sum_{i=1}^{m} \sum_{j=1, j \neq i}^{m} p_{i} p_{j} f_{\mathbb{H}_{\alpha_{i}, \alpha_{j}}^{k_{i}, k_{j}}}(t)
$$

where $f_{\mathbb{H}_{\alpha_{i}, \alpha_{j}}^{k_{i}, k_{j}}}(t)$ is given in $\mathrm{Eq}(16)$ and $f_{\mathbb{E}_{\alpha_{i}}^{2 k_{i}}}(t)$ is given in $\mathrm{Eq}(1)$.

Proof. The proof is a direct consequence of Theorem 3, by taking $\vec{p}=\vec{q}$ and $\vec{k}=\vec{l}$. 


\subsection{Case of Different Rate Parameters}

In this section, we suppose that $X$ and $Y$ are two independent Hyper-Erlang distribution with different rate parameters. We take $X \sim H_{m}(\vec{p}, \vec{\alpha}, \vec{k})$, $\vec{p}=\left(p_{1}, p_{2}, \ldots, p_{m}\right), \vec{\alpha}=\left(\alpha_{1}, \alpha_{2}, \ldots, \alpha_{m}\right)$, and $\vec{k}=\left(k_{1}, k_{2}, \ldots, k_{m}\right)$ and $Y \sim$ $H_{n}(\vec{q}, \vec{\beta}, \vec{l}), \vec{q}=\left(q_{1}, q_{2}, \ldots, q_{n}\right), \vec{\beta}=\left(\beta_{1}, \beta_{2}, \ldots, \beta_{n}\right)$, and $\vec{l}=\left(l_{1}, l_{2}, \ldots, l_{n}\right)$, such that $\alpha_{i} \neq \beta_{j}$ for all $1 \leq i \leq m$ and $1 \leq j \leq n$. From Eq (3) the PDF of $X$ and $Y$ are

$$
f_{X}(t)=\sum_{i=1}^{m} p_{i} f_{\mathbb{E}_{\alpha_{i}}^{k_{i}}}(t) \text { and } f_{Y}(t)=\sum_{j=1}^{n} q_{j} f_{\mathbb{E}_{\beta_{j}}^{l_{j}}}(t)
$$

Theorem 5. Let $X$ and $Y$ are two independent random variables distributed according to (23). Then the PDF of $S=X+Y$ is given by $f_{S}(t)=$ $\sum_{i=1}^{m} \sum_{j=1}^{n} q_{j} p_{i} f_{\mathbb{H}_{\alpha_{i}, \beta_{j}}^{k_{i}, l_{j}}}(t)$, where $f_{\mathbb{H}_{\alpha_{i}, \beta_{j}}^{k_{i}, l_{j}}}(t)$ is given in Eq (16).

Proof. Since $X$ and $Y$ are independent then $\mathcal{L}\left\{f_{S}(t)\right\}=\mathcal{L}\left\{f_{X}(t)\right\} \mathcal{L}\left\{f_{Y}(t)\right\}$ and from Eq (2), $\mathcal{L}\left\{f_{S}(t)\right\}=\sum_{i=1}^{m} \sum_{j=1}^{n} q_{j} p_{i} \frac{\alpha_{i}^{k_{i}}}{\left(s+\alpha_{i}\right)^{k_{i}}} \times \frac{\beta_{j}^{l_{j}}}{\left(s+\beta_{j}\right)^{l_{j}}}$ and so $f_{S}(t)$ is the Laplace inverse of the latter summation. Now, since the case of $X$ and $Y$ of different rate parameters, thus $\alpha_{i} \neq \beta_{j}$ for all $1 \leq i \leq m$ and $1 \leq j \leq n$. Therefore, we obtain from Eq (11) that

$$
f_{S}(t)=\sum_{i=1}^{m} \sum_{j=1}^{n} q_{j} p_{i} f_{\mathbb{H}_{\alpha_{i}, \beta_{j}}^{k_{i}, l_{j}}}(t) .
$$

\subsection{The General Case}

In this section, we consider the general case of the sum of two independent Hyper-Erlang RVs. We suppose that $X$ and $Y$ are two independent HyperErlang distributions with rate parameters not all different. We take $X \sim$ $H_{m}(\vec{p}, \vec{\alpha}, \vec{k})$ and $Y \sim H_{n}(\vec{q}, \vec{\beta}, \vec{l})$ where

1. $\vec{p}=\left(p_{1}, p_{2}, \ldots, p_{m-r}, \tilde{p}_{1}, \tilde{p}_{2}, \ldots, \tilde{p}_{r}\right), \vec{\alpha}=\left(\alpha_{1}, \alpha_{2}, \ldots, \alpha_{m-r}, \gamma_{1}, \gamma_{2}, \ldots, \gamma_{r}\right)$, and $\vec{k}=\left(k_{1}, k_{2}, \ldots, k_{m-r}, \tilde{k}_{1}, \tilde{k}_{2}, \ldots, \tilde{k}_{r}\right)$,

2. $\overrightarrow{\vec{q}}=\left(q_{1}, q_{2}, \ldots, q_{n-r}, \underset{\sim_{1}}{\tilde{q}_{1}} \tilde{q}_{2}, \ldots, \tilde{q}_{r}\right), \vec{\beta}=\left(\beta_{1}, \beta_{2}, \ldots, \beta_{n-r}, \gamma_{1}, \gamma_{2}, \ldots, \gamma_{r}\right)$, and $\vec{l}=\left(l_{1}, l_{2}, \ldots, l_{n-r}, \tilde{l}_{1}, \tilde{l}_{2}, \ldots, \tilde{l}_{r}\right)$. 
We consider that the $r$ rate parameters $\gamma_{1}, \gamma_{2}, \ldots, \gamma_{r}$ are common in $X$ and $Y$ with probability weights $\tilde{p}_{1}, \tilde{p}_{2}, \ldots, \tilde{p}_{r}$ and $\tilde{q}_{1}, \tilde{q}_{2}, \ldots, \tilde{q}_{r}$ respectively and shape parameters $\tilde{k}_{1}, \tilde{k}_{2}, \ldots, \tilde{k}_{r}$ and $\tilde{l}_{1}, \tilde{l}_{2}, \ldots, \tilde{l}_{r}$ respectively, with $\alpha_{i} \neq \beta_{j}$ for all $1 \leq i \leq$ $m-r$ and $1 \leq j \leq n-r$. From Eq (3) the PDF of $X$ and $Y$ can be written as

$$
\begin{aligned}
f_{X}(t) & =\sum_{i=1}^{m-r} p_{i} f_{\mathbb{E}_{\alpha_{i}}^{k_{i}}}(t)+\sum_{i=1}^{r} \tilde{p}_{i} f_{\mathbb{E}_{\gamma_{i}} \widetilde{k_{i}}}(t) \text { and } \\
f_{Y}(t) & =\sum_{j=1}^{n-r} q_{j} f_{\mathbb{E}_{\beta_{j}}^{l_{j}}}(t)+\sum_{j=1}^{r} \tilde{q}_{j} f_{\mathbb{E}_{\gamma_{j}}}(t)
\end{aligned}
$$

Theorem 6. Let $X$ and $Y$ be two independent random variables distributed according to (24) and (25) respectively. Then the PDF of $S=X+Y$ is given by

$$
\begin{aligned}
f_{S}(t)=\sum_{i=1}^{r} \tilde{p}_{i} \tilde{q}_{i} f_{\mathbb{E}_{\bar{k}_{i}+\tilde{l}_{i}}^{\gamma_{i}}}(t)+\sum_{i=1}^{m-r} \sum_{j=1}^{n-r} p_{i} q_{j} f_{\mathbb{H}_{\alpha_{i}, \beta_{j}}^{k_{i}, l_{j}}}(t) \\
+\sum_{i=1}^{m-r} \sum_{j=1}^{r} p_{i} \tilde{q}_{j} f_{\mathbb{H}_{\alpha_{i}, k_{j}}, \tilde{l}_{j}}(t)+\sum_{j=1}^{n-r} \sum_{i=1}^{r} q_{j} \tilde{p}_{i} f_{\mathbb{H}_{\gamma_{i}, \sigma_{j}}, l_{j}}(t)
\end{aligned}
$$

where $f_{\mathbb{H}_{c, d}^{a, b}}(t)$ is given in Eq (16) and $f_{\mathbb{E}_{\bar{k}_{i}+\tau_{i}}^{\gamma_{i}}}(t)$ is given in Eq (1).

Proof. Since $X$ and $Y$ are independent, then

$$
\mathcal{L}\left\{f_{S}(t)\right\}=\mathcal{L}\left\{f_{X}(t)\right\} \mathcal{L}\left\{f_{Y}(t)\right\}
$$

By applying the linear property of Laplace transform in the expression of $f_{X}(t)$ and $f_{Y}(t)$ in $\mathrm{Eq}(24)$ and $(25)$ respectively, we obtain that

$$
\begin{aligned}
& \mathcal{L}\left\{f_{S}(t)\right\} \\
& =\sum_{i=1}^{r} \tilde{p}_{i} \tilde{q}_{i} \mathcal{L}\left\{f_{\mathbb{E}_{\bar{k}_{i}}^{\gamma_{i}}}(t)\right\} \mathcal{L}\left\{f_{\mathbb{E}_{\widetilde{l}_{i}}^{\gamma_{i}}}(t)\right\}+\sum_{i=1}^{m-r} \sum_{j=1}^{n-r} p_{i} q_{j} \mathcal{L}\left\{f_{\mathbb{E}_{\alpha_{i}}^{k_{i}}}(t)\right\} \mathcal{L}\left\{f_{\mathbb{E}_{\beta_{j}}^{l_{j}}}(t)\right\} \\
& +\sum_{i=1}^{m-r} \sum_{j=1}^{r} p_{i} \tilde{q}_{i} \mathcal{L}\left\{f_{\mathbb{E}_{\alpha_{i}}^{k_{i}}}(t)\right\} \mathcal{L}\left\{f_{\mathbb{E}_{\bar{l}_{j}}^{\gamma_{j}}}(t)\right\}+\sum_{j=1}^{n-r} \sum_{i=1}^{r} q_{j} \tilde{p}_{i} \mathcal{L}\left\{f_{\mathbb{E}_{\beta_{j}}^{l_{j}}}(t)\right\} \mathcal{L}\left\{f_{\mathbb{E}_{\bar{k}_{i}}^{\gamma_{i}}}(t)\right\} .
\end{aligned}
$$

However all of the Laplace transforms in the above expression are of Erlang distributions, are given in $\mathrm{Eq}(2)$. Hence, we can write $\mathcal{L}\left\{f_{S}(t)\right\}$ as 


$$
\begin{aligned}
& \sum_{i=1}^{r} \tilde{p}_{i} \tilde{q}_{i} \frac{\gamma_{i}^{\widetilde{k_{i}}+\widetilde{l_{i}}}}{\left(s+\gamma_{i}\right)^{\widetilde{k_{i}}+\widetilde{l}_{i}}}+\sum_{i=1}^{m-r} \sum_{j=1}^{n-r} p_{i} q_{j} \frac{\alpha_{i}^{k_{i}}}{\left(s+\alpha_{i}\right)^{k_{i}}} \frac{\beta_{j}^{l_{j}}}{\left(s+\beta_{j}\right)^{l_{i}}} \\
& \quad+\sum_{i=1}^{m-r} \sum_{j=1}^{r} p_{i} \tilde{q}_{j} \frac{\alpha_{i}^{k_{i}}}{\left(s+\alpha_{i}\right)^{k_{i}}} \frac{\gamma_{j}^{\widetilde{l_{j}}}}{\left(s+\gamma_{j}\right)^{\widetilde{l_{j}}}}+\sum_{j=1}^{n-r} \sum_{i=1}^{r} q_{j} \tilde{p}_{i} \frac{\beta_{j}^{l_{j}}}{\left(s+\beta_{j}\right)^{l_{i}}} \frac{\gamma_{i}^{\widetilde{k_{i}}}}{\left(s+\gamma_{i}\right)^{\widetilde{k_{i}}}}
\end{aligned}
$$

Now, $f_{S}(t)$ is the Laplace inverse of Eq (26), and again by applying the property of linearity for Laplace inverse transforms, we only need the Laplace inverse of the fractions in Eq (26). From Eq (2), we have

$$
\mathcal{L}^{-1}\left\{\frac{\gamma_{i}^{\widetilde{k_{i}}+\widetilde{l_{i}}}}{\left(s+\gamma_{i}\right)^{\widetilde{k_{i}}+\widetilde{l_{i}}}}\right\}=f_{\mathbb{E}_{\widetilde{k_{i}}+\widetilde{l_{i}}}^{\gamma_{i}}}(t)
$$

and the other fractions has the form of two partial distinct fractions since $\alpha_{i}, \beta_{j}$ and $\gamma_{u}$ are different for all $1 \leq i \leq m-r, 1 \leq j \leq n-r$ and $1 \leq u \leq r$. Therefore, we can refer to Eq (10), that the Laplace inverse of these fractions is the PDF of the sum of two Erlangs, and we may conclude that

$$
\begin{aligned}
f_{S}(t)=\sum_{i=1}^{r} \tilde{p}_{i} \tilde{q}_{i} f_{\mathbb{E}_{\bar{k}_{i}+\tilde{l}_{i}}^{\gamma_{i}}}(t) & +\sum_{i=1}^{m-r} \sum_{j=1}^{n-r} p_{i} q_{j} f_{\mathbb{H}_{\alpha_{i}, \beta_{j}} k_{i}, l_{j}}(t) \\
& +\sum_{i=1}^{m-r} \sum_{j=1}^{r} p_{i} \tilde{q}_{j} f_{\mathbb{H}_{\alpha_{i}, \gamma_{j}}^{k_{i}, \widetilde{l}_{j}}}(t)+\sum_{j=1}^{n-r} \sum_{i=1}^{r} q_{j} \tilde{p}_{i} f_{\mathbb{H}_{\gamma_{i}, \beta_{j}} \widetilde{k}_{i}, l_{j}}(t) .
\end{aligned}
$$

\section{Conclusion}

The exact expressions of the PDF of the sum of two independent Hyper-Erlang distributions are given in all its possible cases. The problem is solved by finding a closed exact expression of $A_{i j}$, the coefficient of the linear combination in Eq (6), and then finding an exact expression of PDF for the sum of two independent Erlang distributions is determined, that was proved to be a function of the Kummer confluent hypergeometric function, ${ }_{1} F_{1}[a, b, z]$.

\section{References}

[1] A. S Alfa . and W Li, A homogeneous PCS network with markov call arrival process and phase type cell residence time. Wireless Networks, 8, No.6 (2002), 597-605. 
[2] L.C Andrews, Special Functions of Mathematics for Engineers, 2nd ed., McGraw-Hill, New York (1992).

[3] F. Barcelo, J. Jordan, Channel holding time distribution in cellular telephony, In Proc. 9th Int. Conf. on Wireless Communications, 1, Calgary, AB, Canada, July, 9-11,(1997), 125-134. doi:10.1049/el:19980165/

[4] G. Bolch, S. Greiner, H. Meer, K. Trivedi, Queuing Networks and Markov Chains: Modeling and Performance Evaluation with Computer Science Applications, John Wiley \& Sons (2006).

[5] T. K. Christensen , B. F. Nielsen, V. B. Iversen, Phase-type models of channel-holding times in cellular communication systems, IEEE Trans. Veh. Technol., 53, No. 3 (2004),725 -733.

[6] Y. Fang, Analytical Generalized Results for Handoff Probability in Wireless Networks, IEEE Transactions on Communications, 50, No. 3 (2002), 396-399. .

[7] Y. Fang, I. Chlamtac, Y.B. Lin, Modeling PCS Networks Under General Call Holding Times and Cell Residence Time Distributions, IEEE Trans. Networking, 5, No. 6 (1997), 893-906.

[8] Y. G. Fang, I Chlamtac, Teletraffic analysis and mobility modeling of PCS networks, IEEE Trans. Commun., 47, No. 7 (1999), 1062-1072.

[9] W. Feller, An Introduction to Probability Theory and Its Applications, Vol. II. John Wiley \& Sons, New York (1971).

[10] S. Glisic, B. Lorenzo, Advanced Wireless Networks: 4 G Cognitive Opportunistic and Cooperative Technology, 2nd ed John Wiley \& Sons, 2009.

[11] J. L. Gross, Combinatorial Methods with Computer Applications, Boca Raton,FL: Chapman \& Hall/CRC (2008).

[12] H. Jasiulewicz, W. Kordecki, Convolutions of Erlang and of Pascal distributions with applications to reliability, Demonstratio Math, 36, No. 1 (2003), 231-238.

[13] C. Jedrzycki, V.C. Leung, Probability distribution of channel holding time in cellular telephony systems, In Proceedings of IEEE VTC'96 Vehicular Technology Conference, 1 (1996), 247-251. doi: 10.1109/VETEC.1996.503446 
[14] J. Jordan, F. Barcelo, Statistical modeling of channel occupancy in trunked PAMR systems, In Proc. 15th Int. Teletraffic Conf., (1997), 1169-1178.

[15] T. Kadri, K. Smaili, The Exact Distribution of The Ratio of Two Independent Hypoexponential Random Variables, British Journal of Mathematics \& Computer Science, 4, No.18 (2014), 2665-2675. doi: 10.9734/BJMCS/2014/11217.

[16] T. Kadri, K. Smaili, S. Kadry, Markov Modeling for Reliability Analysis Using Hypoexponential Distribution. In S. Kadry, A. El Hami (Ed.), Numerical Methods for Reliability and Safety Assessment, Springer, New York, USA, (2014).

[17] S. Nadarajah, A review of results on sums of random variables, Acta Applicandae Mathemematicae, 103, No. 2 (2008), 131-140. doi:10.1007/s10440008-9224-4.

[18] P.V. Orlik and S.S. Rappaport, A model for teletraffic performance and channel holding time characterization in wireless cellular communication with general session and dwell time distributions, IEEE J. Select. Areas Commun., 16, Vol. 5 (1998), 788-803. doi: 10.1109/49.700913.

[19] P.V. Orlik and S. S. Rappaport, Traffic performance and mobility modeling of cellular communications with mixed platforms and highly variable mobilities, In Proc. of the IEEE, 86, No. 7 (1998), 1464-1479. doi: $10.1109 / 5.681374$.

[20] S. M. Ross, Introduction to Probability Models, 10th ed, Academic Press, San Diego (2011).

[21] K. Smaili, T. Kadri, S. Kadry, A Modified-Form Expressions For The Hypoexponential Distribution, British Journal of Mathematics \& Computer Science, 4, No. 3 (2014), 322-332. doi: 10.9734/BJMCS/2014/6317.

[22] K. Smaili, T. Kadri, S. Kadry, Hypoexponential Distribution with Different Parameters, Applied Mathematics, 4, No. 4 (2013), 624-631. doi: 10.4236/am.2013.44087.

[23] B. H. Soong, J. A. Barria, A Coxian model for channel holding time distribution for teletraffic mobility modeling, IEEE Commun. Lett., 4, No. 12 (2000), 402 -404. doi: 10.1109/4234.898721 
[24] K.S Trivedi, Probability and Statistics with Reliability, Queuing and Computer Science Applications, 2nd ed, John Wiley \& Sons, (2002)

[25] H. Zeng and I. Chlamtac, Handoff traffic distribution in cellular networks, In Proceedings of IEEE WCNC'99 Wireless Communications and Networking Conference, 1, (1999), 413-417. doi: 10.1109/WCNC.1999.797858.

[26] H. Zeng, Y. Fang, I. Chlamtac, Call blocking performance study for PCS networks under more realistic mobility assumptions, Telecommunication Systems, 19, No.2 (2002), 125-146.

[27] M. M. Zonoozi, P. Dassanayake, User mobility modeling and characterization of mobility patters, IEEE J. Select. Areas Commun., 15, No. 7 (1997), 1239-1252. doi: 10.1109/49.622908.

[28] M. M. Zonoozi , P. Dassanayake, M. Faulkner, Mobility modelling and channel holding time distribution in cellularmobile communication systems, In Proc. of IEEE GLOBECOM'95, Global Telecommunications Conference, 1, (1995), 12-16.

[29] Wolfram Function site (2014), Kummer confluent hypergeometric function, see http://functions.wolfram.com/07.20.03.0119.01. 